Pacific Journal of Mathematics

QUASICOMPACTIFICATIONS AND SHAPE THEORY 


\title{
QUASICOMPACTIFICATIONS AND SHAPE THEORY
}

\author{
B. J. BALL
}

If $f: X \rightarrow Y$ is an embedding of a space $X$ into a space $Y$ such that each component of $Y$ is a compactification of the image of a quasicomponent of $X$ and such that $f$ induces a homeomorphism of the space $Q X$ of quasicomponents of $X$ onto the space of components of $Y$, then $(f, Y)$ is called a quasicompactification of $X$. After some preliminary results, it is shown that a locally compact metric space $X$ has a locally compact metric quasicompactification if and only if $Q X$ is locally compact. Two canonical quasicompactifications, $F^{*} X$ and $\alpha X$, of such a space are described, and it is shown that if $\operatorname{Sh}_{p} X=\operatorname{Sh}_{p} Y$, then $\operatorname{Sh}_{p} F^{*} X=\mathrm{Sh}_{p} F^{*} Y$; the question, whether also $\operatorname{Sh}_{p} \alpha X=\mathrm{Sh}_{p} \alpha Y$ is left open. Finally, some techniques of this paper are used to obtain a proper shape version of a theorem due to Y. Kodama, generalizing previous work of the author.

1. Introduction. A subset $A$ of a topological space $X$ is a component of $X$ if it is maximal with respect to the property that no two points of $A$ are separated in the subspace $A$, and is a quasicomponent of $X$ if it is maximal with respect to the property that no two points of $A$ are separated in the whole space $X$. Thus quasicomponents are more dependent on, and indicative of, the global structure of $X$ than are components.

It is easily shown that both the set $\mathscr{C}_{X}$ of all components of $X$ and the set $q \mathscr{C}_{X}$ of all quasicomponents of $X$ are decompositions of $X$ into disjoint closed sets. The resulting spaces $X / \mathscr{C}_{X}$ and $X / q \mathscr{C}_{X}$ (with the decomposition topologies) are not in general very nice spaces; for example, even if $X$ is separable and metric, $X / \mathscr{C}_{X}$ need not be Hausdorff and $X / q \mathscr{C}_{X}$, while always Hausdorff, need not have a basis of open and closed sets (see [12]). Retopologizing $X / q \mathscr{C}_{X}$, however, avoids this latter difficulty; specfically, the quasicomponent space of $X$ is the space $Q X$ whose points are the quasicomponents of $X$ and whose topology has as a basis those sets of quasicomponents whose union is both open and closed in $X$. The space $Q X$ thus has a basis consisting of open and closed sets (i.e., $Q X$ is 0dimensional) and hence is regular and totally disconnected.

Elementary, well known arguments suffice to establish the following useful facts. If $p$ is a point of a topological space $X$, the component of $X$ about $p$ is the union of all connected subsets of $X$ containing $p$, and the quasicomponent of $X$ about $p$ is the intersection of all open and closed subsets of $X$ containing $p$. Hence 
any component of $X$ contains every connected subset of $X$ which intersects it, and every quasicomponent of $X$ is contained in every open and closed subset of $X$ which intersects it.

Any two components of a compact Hausdorff space $X$ are separated in $X$, but this fails in the absence of compactness (even if $X$ is locally compact and metrizable). However, any two quasicomponents of any topological space $X$ are separated in $X$.

For a locally compact Hausdorff space $X$, any compact component of $X$ is a quasicomponent of $X$. Combining this fact with other well known results gives the following theorem.

THEOREM 1.1. If $X$ is a locally compact Hausdorff space such that every component of $X$ is compact, then $\mathscr{C}_{X}=q \mathscr{C}_{X}, \mathscr{C}_{X}$ is upper semicontinuous, $X / \mathscr{C}_{X}$ is locally compact and totally disconnected (and is metrizable if $X$ is), and $Q X=X / q \mathscr{C}_{X}=X / \mathscr{C}_{X}$.

This observation suggests that it might be desirable, when possible, to construct a "nice" embedding of a given space $X$ into a space $Y$ which has compact components. In this paper it will be shown how this may be done for certain spaces, and some shapetheoretic properties of the resulting "quasicompactifications" will be considered.

2. Definitions and preliminary results. If $\left\{X_{\alpha} \mid \alpha \in A\right\}$ is a collection of disjoint, nonempty topological spaces (with $X_{\alpha} \neq X_{\beta}$ for $\alpha \neq \beta$ ), then $\cup X_{\alpha}$, with the topology in which a set is open if and only if its intersection with each $X_{\alpha}$ is open in $X_{\alpha}$, is called the topological sum of the $X_{\alpha}$ 's and is denoted by $\oplus\left\{X_{\alpha} \mid \alpha \in A\right\}$ or by $\oplus$ $X_{\alpha}$. Equivalently, a space $X$ is the topological sum of subspaces $\left\{X_{\alpha}\right\}$ if the $X_{\alpha}$ 's are disjoint, nonempty, open and closed subsets of $X$ and $\cup X_{\alpha}=X$.

LEMma 2.1. If $X=\bigoplus\left\{X_{\alpha} \mid \alpha \in A\right\}$, then $Q X=\bigoplus\left\{Q X_{\alpha} \mid \alpha \in A\right\}$; conversely, if $Q X=\bigoplus\left\{Z_{\alpha} \mid \alpha \in A\right\}$, then $X=\oplus\left\{X_{\alpha} \mid \alpha \in A\right\}$ with $Q X_{\alpha}=$ $Z_{\alpha}$ for each $\alpha$.

Proof. The first assertion follows immediately from the fact that if $K$ is open and closed in $X$, then $Q K$ is open and closed in $Q X$. For the converse, let $p: X \rightarrow Q X$ be the natural projection and let $X_{\alpha}=p^{-1}\left(Z_{\alpha}\right)$ for each $\alpha$ in $A$. It is clear that $X=\oplus X_{\alpha}$ and that $Q X_{\alpha}=Z_{\alpha}$ for each $\alpha$.

LEMMA 2.2. If $X$ is a locally compact metrizable space, then $Q X$ is a Lindelöf space (i.e., every open cover has a countable subcover) if and only if $X$ is separable. 
Proof. If $X$ is separable, then $Q X$ is a Lindelöf space since the Lindelof property is preserved by continuous surjective maps.

For the converse, suppose $Q X$ has the Lindelöf property and write $X$ in the form $X=\bigoplus\left\{X_{\alpha} \mid \alpha \in A\right\}$ with each $X_{\alpha}$ separable (see [5], p. 241, Th. 7.3). Then $Q X=\bigoplus\left\{Q X_{\alpha} \mid \alpha \in A\right\}$ and since $Q X$ has the Lindelöf property, it follows that $A$ is countable. Hence $X$ is the union of a countable number of separable spaces and hence is separable.

LEMMA 2.3. If $X$ is a locally compact metrizable space and $Q X$ is compact, then $Q X$ is metrizable.

Proof. It follows from Lemma 2.2 that $X$ is separable. Since any compact Hausdorff space which is the continuous image of a separable metric space is metrizable ([8], p. 115, Lemma 40), it follows that $Q X$ is metrizable.

LEMMA 2.4. If $X$ is a locally compact metric space, then $Q X$ is locally compact if and only if $X=\bigoplus\left\{X_{\alpha} \mid \alpha \in A\right\}$ with $Q X_{\alpha}$ compact for each $\alpha$.

Proof. Since, as observed before, any locally compact metric space can be written as the topological sum of separable spaces, it is sufficient to prove the result for the case in which $X$ is separable. One direction follows immediately from Lemma 2.1; for the converse, suppose $Q X$ is locally compact and $X$ is separable. By Lemma 2.2, $Q X$ is a Lindelöf space and, since $Q X$ is 0 -dimensional, it easily follows that $Q X=\bigoplus\left\{Z_{n} \mid n=1,2, \cdots\right\}$ with $Z_{n}$ compact for each $n$. By Lemma 2.1, $X=\bigoplus\left\{X_{n} \mid n=1,2, \cdots\right\}$ with $Q X_{n}=Z_{n}$ for each $n$. The theorem follows.

It is clear that for any map (i.e., continuous function) $f: X \rightarrow Y$, the image under $f$ of any quasicomponent of $X$ lies in a (unique) quasicomponent of $Y$.

Lemma 2.5. If $f: X \rightarrow Y$ and for each $A \in Q X, \phi_{f}(A)$ is the quasicomponent of $Y$ containing $f(A)$, then $\phi_{f}$ is a continuous function from $Q X$ to $Q Y$.

Proof. Let $\mathscr{C}$ be a basic open set in $Q Y$. Then $\cup \mathscr{C}$ is open and closed in $Y$ and hence $f^{-1}(\cup \mathscr{C})$ is open and closed in $X$. If $\mathscr{T}=$ $\left\{A \in Q X \mid A \subset f^{-1}(\cup \mathscr{C})\right\}$, then $\cup \mathscr{V}=f^{-1}(\cup \mathscr{C})$, so $\mathscr{V}$ is open in $Q X$. Using the fact that $\cup \mathscr{C}$ and $\cup \mathscr{Y}$ are open and closed in $Y$ and $X$, respectively, it is easily shown that $\phi_{f}^{-1}(\mathscr{C})=\mathscr{V}$. Thus $\phi_{f}$ is continuous. 
The map $\phi_{f}$ described in Lemma 2.2 will be called the map of $Q X$ into $Q Y$ induced by the map $f: X \rightarrow Y$.

3. Quasicompactifications. Throughout the remainder of this paper, unless the contrary is specifically indicated all spaces considered are assumed to be locally compact and metrizable. If $X$ is such a space, then a quasicompactification of $X$ is a pair $(Y, f)$ such that $Y$ is a (locally compact metrizable) space with compact components and $f: X \rightarrow Y$ is an embedding satisfying (1) for each $A$ in $Q X, \operatorname{cl}_{Y} f(A)$ is a component of $Y$ and (2) the map $\phi_{f}: Q X \rightarrow Q Y$ induced by $f$ is a homeomorphism. (Here, as usual, $\operatorname{cl}_{Y} f(A)$ denotes the closure of $f(A)$ in $Y$.) Following the usual practice with respect to compactifications, we will often call the space $Y$ a quasicompactification of $X$, considering $X$ as a subspace of $Y$ with $f: X \rightarrow Y$ the inclusion map.

Note that condition (1) alone implies that $\phi_{f}$ is a continuous bijection of $Q X$ onto a subspace of $Q Y$. In general, however, $\phi_{f}$ need not be a homeomorphism, even if it is surjective.

In order that $X$ should have a quasicompactification, it is clearly necessary that $Q X$ be locally compact since $Q X \approx Q Y$ and $Q Y$ is locally compact by Theorem 1.1. Local compactness of $Q X$ is also a sufficient condition for $X$ to have a quasicompactification, as the following lemmas show.

Recall that the Freudenthal compactification $F X$ of a rim-compact space $X$ is characterized by the property that no open neighborhood of a point $p$ of $E X(=F X-X)$ is separated by $E X$ into two disjoint open sets each having $p$ as a limit point. (The definition of $F X$ and additional properties of this compactification may be found in [6], [11] and [8]; additional references and a characterization of $F X$ in terms of nonconvergent sequences, for $X$ locally compact and metrizable and $Q X$ compact, are given in [2].)

Lemma 3.1. The closure in FX of any open and closed subset of $X$ is open and closed in $F X$.

This is an easy consequence of the characterization of $F X$ quoted above.

THEOREM 3.2. If $Q X$ is compact, then $F X$ is a quasicompactification of $X$.

Proof. It is well known that in this case $F X$ is metrizable. Considering $X$ as a subspace of $F X$ and letting $f: X \rightarrow F X$ be the inclusion map, we must show that conditions (1) and (2) of the definition are satisfied. 
First suppose $A$ is a quasicomponent of $X$ and let $C$ be the component of $F X$ containing $A$. By Lemma 3.1, any two points of $X$ which are separated in $X$ are separated in $F X$ and hence $C-A \subset$ $E X$. Since $C$ is connected and $E X$ is totally disconnected, it follows that $C=\operatorname{cl}_{F X} A$. Hence condition (1) holds, and it remains only to show that the induced map $\phi\left(=\phi_{f}\right)$ is surjective and open.

To see that $\phi$ is surjective, it is sufficient to show that each component $C$ of $F X$ intersects $X$, for then $C$ contains some $A \in Q X$ and, by the argument above, $\mathrm{cl}_{F X} A=C=\phi(A)$. If $C$ is a component of $F X$ which does not intersect $X$, then $C=\{p\}$ for some point $p \in$ $E X$. Since $X$ is dense in $F X$ (and $F X$ is metrizable), there is a sequence $\alpha=\left(x_{1}, x_{2}, \cdots\right)$ of points of $X$ converging to $p$ in $F X$. If any quasicomponent $A$ of $X$ contains infinitely many points of $\alpha$, then $p \in \operatorname{cl}_{F X} A \subset C$ and hence $A \subset C$, contrary to the assumption that $C \cap X=\varnothing$. Hence suppose that no two points of $\alpha$ belong to the same quasicomponent of $X$, and for each $i$, let $A_{i}$ be the quasicomponent of $X$ containing $x_{i}$. Since $Q X$ is compact, it is metrizable by Lemma 2.3 and hence some subsequence of $\left\{A_{i}\right\}$ converges in $Q X$; suppose, without loss, that $\left\{A_{i}\right\} \rightarrow A \in Q X$. Then $p \notin \mathrm{cl}_{F X} A$, so $F X=$ $H \cup K$, with $H$ and $K$ closed and disjoint, $A \subset H$ and $p \in K$. Since $A_{i} \rightarrow A$ in $Q X$ and $H \cap X$ is an open and closed subset of $X$ containing $A, A_{i} \subset H \cap X$ for almost all $i$; but $x_{i} \in K$ for almost all $i$ since $x_{i} \rightarrow$ $p \in K$, and hence $A_{i} \cap K \neq \varnothing$ for almost all $i$. This is a contradiction, and it follows that every component of $F X$ intersects $X$, so $\phi$ is surjective.

Now suppose $\mathscr{C}$ is a basic open set in $Q X$. Then $U=U \mathscr{C}$ is open and closed in $X$, so $\operatorname{cl}_{F X} U$ is open and closed in $F X$. If $p \in$ $\operatorname{cl}_{F X} U$, then by the preceding argument, $p \in \operatorname{cl}_{F X} A$ for some $A \in Q X$. Since $U$ is open and closed in $X$, this implies that

$$
\operatorname{cl}_{F X} U=\cup\left\{\mathrm{cl}_{F X} A \mid A \subset U\right\} \text {. }
$$

Since $\phi(\mathscr{C})=\left\{\mathrm{cl}_{F X} A \mid A \subset U\right\}$ and $U\left\{\mathrm{cl}_{F X} A \mid A \subset U\right\}$ is open and closed in $F X, \phi(\mathscr{U})$ is open (and closed) in $Q F X$. Hence $\phi$ is an open map, and since it is $1-1$ and onto, $\phi: Q X \rightarrow Q F X$ is a homeomorphism. Thus $F X$ is a quasicompactification of $X$.

CoROLlary 3.3. If $Q X$ is locally compact, then $X$ has a quasicompactification.

Proof. By Lemma 2.4, $X=\bigoplus\left\{X_{\alpha} \mid \alpha \in A\right\}$ with $Q X_{\alpha}$ compact for each $\alpha$. By Theorem 3.2, FX $X_{\alpha}$ is a quasicompactification of $X_{\alpha}$ and it readily follows that $\bigoplus\left\{F X_{\alpha} \mid \alpha \in A\right\}$ is a quasicompactification of $X$.

The following theorem shows that the quasicompactification of 
$X$ described in the proof of Corollary 3.3 is independent of the choice of the $X_{\alpha}$ 's; this quasicompactification will be denoted by $F^{*} X$ and will be called the Freudenthal quasicompactification of $X$.

Theorem 3.4. If $X=\bigoplus\left\{X_{\alpha} \mid \alpha \in A\right\}$ and $X=\bigoplus\left\{Y_{\beta} \mid \beta \in B\right\}$, with $Q X_{\alpha}$ and $Q Y_{\beta}$ compact for $\alpha \in A$ and $\beta \in B$, respectively, then $\bigoplus\left\{F X_{\alpha} \mid \alpha \in A\right\}$ is homeomorphic to $\oplus\left\{F Y_{\beta} \mid \beta \in B\right\}$.

Proof. We first observe that if $Z$ is a space such that $Q Z$ is compact and $Z=\bigoplus\left\{Z_{\tau} \mid \tau \in T\right\}$, then $F Z=\bigoplus\left\{F Z_{\tau} \mid \tau \in T\right\}$. To see this, note that by Lemma 2.1, $Q X=\bigoplus\left\{Q Z_{\tau} \mid \tau \in T\right\}$, and since $Q Z$ is compact, $T$ must be finite. Hence $\bigoplus\left\{F Z_{\tau} \mid \tau \in T\right\}$ is a compactification of $Z$. Using the characterization of the Freudenthal compactification given earlier, it is easily shown that $\oplus\left\{F Z_{\tau} \mid \tau \in T\right\}=F Z$.

Now for each $\alpha \in A, X_{\alpha}=\bigoplus\left\{X_{\alpha} \cap Y_{\beta} \mid \beta \in B, X_{\alpha} \cap Y_{\beta} \neq \varnothing\right\}$ so, by the previous remark, $F X_{\alpha}=\bigoplus\left\{F\left(X_{\alpha} \cap Y_{\beta}\right) \mid \beta \in B, X_{\alpha} \cap Y_{\beta} \neq \varnothing\right\}$; therefore $\bigoplus\left\{F X_{\alpha} \mid \alpha \in A\right\}=\bigoplus\left\{F\left(X_{\alpha} \cap X_{\beta}\right) \mid \alpha \in A, \beta \in B, X_{\alpha} \cap Y_{\beta} \neq \varnothing\right\}$. Similarly $\bigoplus\left\{F Y_{\beta} \mid \beta \in B\right\}=\bigoplus\left\{F\left(Y_{\beta} \cap X_{\alpha}\right) \mid \beta \in B, \alpha \in A, Y_{\beta} \cap X_{\alpha} \neq \varnothing\right\}$, and hence $\bigoplus\left\{F X_{\alpha} \mid \alpha \in A\right\}=\bigoplus\left\{F Y_{\beta} \mid \beta \in B\right\}$.

Simple examples show it is not the case that each component of the Freudenthal quasicompactification of $X$ is necessarily the Freudenthal compactification of a quasicomponent of $X$. As the next theorem shows, however, if $X$ has a quasicompactification at all, it has one in which each component is the Alexandroff compactification of a quasicomponent of $X$. (Here, the Alexandroff compactification of a space $Z$ is the one-point compactification if $Z$ is not compact, and is $Z$ itself if $Z$ is compact.)

THEOREM 3.5. If $Q X$ is locally compact, there is a topologically unique quasicompactification $Y$ of $X$ such that each component of $Y$ is the Alexandroff compactification of a quasicomponent of $X$.

Proof. For each quasicomponent $A$ of $X$, let $\bar{A}$ denote the closure of $A$ in $F^{*} X$ and let $\mathscr{G}=\left\{\bar{A}-A \mid A \in q \mathscr{C}_{X}\right\}$. It is easy to see that $\mathscr{G}$ is an upper semicontinuous collection of disjoint closed subsets of $F^{*} X$. If $Y=F X / \mathscr{G}$ and $p: F^{*} X \rightarrow Y$ is the projection map, it is clear that for each $A \in Q X, p(\bar{A})$ is the Alexandroff compactification of $A$. Since no element of $\mathscr{G}$ intersects two components of $F^{*} X$, it easily follows that the components of $Y$ are precisely the sets $p(A)$ for $A \in Q X$.

By definition, the map $\phi: Q X \rightarrow Q\left(F^{*} X\right)$ defined by $\phi(A)=\bar{A}$ for $A \in Q X$ is a homeomorphism. Since the map $\psi: Q\left(F^{*} X\right) \rightarrow Q Y$ defined by $\psi(\bar{A})=p(\bar{A})$ is also a homeomorphism, so is the map $\psi \circ \phi: Q X \rightarrow$ $Q Y$ and it follows that $Y$ is a quasicompactification of $X$. 
To see that $Y$ is topologically unique, suppose $Y^{\prime}$ is any quasicompactification of $X$ with each component of $Y^{\prime}$ the Alexandroff compactification of a quasicomponent of $X$; for simplicity, assume that $X \subset Y^{\prime}$. For each noncompact quasicomponent $A$ of $X$, let $p_{A}=\left(\mathrm{cl}_{Y} A\right)-A$ and $p_{A}^{\prime}=\left(\mathrm{cl}_{Y^{\prime}} A\right)-A$. If $h: Y \rightarrow Y^{\prime}$ is defined by $h(x)=x$ for $x \in X$ and $h\left(p_{A}\right)=p_{A}^{\prime}$ for $A$ a noncompact quasicomponent of $X$, then $h$ is a homeomorphism of $Y$ onto $Y^{\prime}$.

The quasicompactification $Y$ described in the above proof will be called the Alexandroff quasicompactification of $X$, and will be denoted by $\alpha X$.

4. Shape properties. For an arbitrary topological space $X$, we denote the shape of $X$ in the sense of Mardešic [10] by Sh $X$; if $X$ is locally compact and metrizable, $\mathrm{Sh}_{p} X$ will denote the proper shape of $X$ in the sense of [4] and $\mathrm{Sh}_{p}^{1} X$ the proper shape of $X$ in the alternative sense described in [3]. The following "decomposition theorem", which was essentially proved in [4], probably has analogues for $\mathrm{Sh}_{p}^{1} X$ and for $\mathrm{Sh} X$, though this is by no means clear (to the present author, at least).

THEOREM 4.1. If $X$ and $Y$ are locally compact metric spaces and $X=\oplus\left\{X_{\alpha} \mid \alpha \in A\right\}$, then $\operatorname{Sh}_{p} X=\operatorname{Sh}_{p} Y$ (respectively, $\operatorname{Sh}_{p} X \leqq \operatorname{Sh}_{p} Y$ ) if and only if $Y=\bigoplus\left\{Y_{\alpha} \mid \alpha \in A\right\}$ with $\mathrm{Sh}_{p} X_{\alpha}=\mathrm{Sh}_{p} Y_{\alpha}$ (resp., $\mathrm{Sh}_{p} X_{\alpha} \leqq$ $\mathrm{Sh}_{p} Y_{\alpha}$ ) for each $\alpha \in A$.

Proof. It follows from Lemma 5.5 of [4] and from the proof of Lemma 5.8 of the same paper that $\mathrm{Sh}_{p} X=\mathrm{Sh}_{p} Y$ if and only if there exist locally compact ANR's $P$ and $Q$ containing $X$ and $Y$, respectively, as closed subsets, and proper fundamental nets $\underline{f}: X \rightarrow Y$

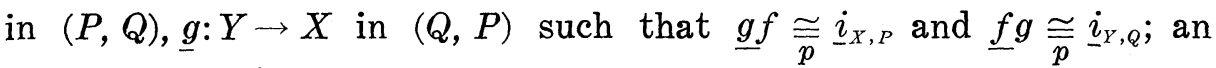

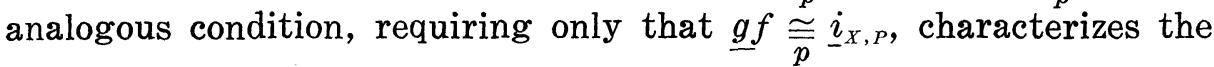
relation $\mathrm{Sh}_{p} X \leqq \mathrm{Sh}_{p} Y$. It follows immediately that if $X=\oplus$ $\left\{X_{\alpha} \mid \alpha \in A\right\}$ and $Y=\bigoplus\left\{Y_{\alpha} \mid \alpha \in A\right\}$ with $\mathrm{Sh}_{p} X_{\alpha}=\mathrm{Sh}_{p} Y_{\alpha}$ (respectively, $\mathrm{Sh}_{p} X_{\alpha} \leqq \mathrm{Sh}_{p} Y_{\alpha}$ ) for each $\alpha \in A$, then $\mathrm{Sh}_{p} X=\mathrm{Sh}_{p} Y$ (respectively, $\left.\mathrm{Sh}_{p} X_{\alpha} \leqq \operatorname{Sh}_{p} Y_{\alpha}\right)$.

For the converse, suppose $\mathrm{Sh}_{p} X=\mathrm{Sh}_{p} Y$ (respectively, $\mathrm{Sh}_{p} X \leqq$ $\left.\mathrm{Sh}_{p} Y\right)$ and $X=\bigoplus\left\{X_{\alpha} \mid \alpha \in A\right\}$. As above, there exist locally compact ANR's $P$ and and $Q$ containing $X$ and $Y$, respectively, as closed subsets, and proper fundamental nets $\underline{f}: X \rightarrow Y$ in $(P, Q), \underline{g}: Y \rightarrow X$ in $(Q, P)$ such that $g f \cong \underline{\bar{i}}_{X, P}$ and $\underline{f g} \cong \underline{\bar{i}}_{Y, Q}$ (or only the first of these if $\mathrm{Sh}_{p} X \leqq \mathrm{Sh}_{p} Y$ ). Theorem 5.2 of [4] shows that $Y$ can be written 
as $Y=\bigoplus\left\{Y_{\alpha} \mid \alpha \in A\right\}$ with $\underline{g f} \cong \widetilde{\bar{p}}_{X_{\alpha}, P}$ and $\underline{f} g \underset{\bar{p}}{\cong} \underline{i}_{Y_{\alpha}, Q}$ (or only the first of these, if $\operatorname{Sh}_{p} X \leqq \operatorname{Sh}_{p} Y$ ) for each $\alpha \in A$. It follows that $\operatorname{Sh}_{p} X_{\alpha}=$ $\mathrm{Sh}_{p} Y_{\alpha}$ (resp., $\mathrm{Sh}_{p} X_{\alpha} \leqq \mathrm{Sh}_{p} Y_{\alpha}$ ), as required.

Lemma 4.2. If $\mathrm{Sh}_{p} X \leqq \mathrm{Sh}_{p} Y$ and $Q X$ is compact, then $Q Y$ is compact.

Proof. Lemma 2.2 implies that $X$ is separable and hence ([4], Lemma 5.3) $Y$ is also separable. By Lemma 2.2, $Q Y$ is a Lindelöf space. If $Q Y$ is not compact then, since it is 0-dimensional, $Q Y=$ $\bigoplus Z_{n}$, where $Z_{1}, Z_{2}, \cdots$ is an infinite set of disjoint (nonempty) open subsets of $Q Y$. By Lemma 2.1, $Y=\bigoplus Y_{n}$ with $Q Y_{n}=Z_{n}$ for each $n$, and by Theorem 4.1, $X=\bigoplus X_{n}$ with $\operatorname{Sh}_{p} X_{n} \leqq \operatorname{Sh}_{p} Y_{n}$ for each $n$. But then $Q X=\bigoplus Q X_{n}$, and this is impossible since $Q X$ is compact and therefore cannot be the union of infinitely many disjoint open sets.

THEOREM 4.3. If $\mathrm{Sh}_{p} X=\mathrm{Sh}_{p} Y$ and $Q X$ is locally compact, then $Q Y$ is locally compact and $\operatorname{Sh}_{p} F^{*} X=\operatorname{Sh}_{p} F^{*} Y$.

Proof. By Lemma 2.4, $X=\bigoplus\left\{X_{\alpha} \mid \alpha \in A\right\}$ with each $Q X_{\alpha}$ compact. By Theorem 4.1, $Y=\bigoplus\left\{Y_{\alpha} \mid \alpha \in A\right\}$ with $\operatorname{Sh}_{p} X_{\alpha}=\operatorname{Sh}_{p} Y_{\alpha}$ for each $\alpha$; since $Q X_{\alpha}$ is compact, $Q Y_{\alpha}$ is compact by Lemma 4.2 and since $Q Y=$ $\bigoplus\left\{Q Y_{\alpha} \mid \alpha \in A\right\}, Q Y$ is locally compact. By definition, $F^{*} X=$ $\bigoplus\left\{F X_{\alpha} \mid \alpha \in A\right\}$ and $F^{*} Y=\bigoplus\left\{F Y_{\alpha} \mid \alpha \in A\right\}$; since $\mathrm{Sh}_{p} X_{\alpha}=\mathrm{Sh}_{p} Y_{\alpha}$, Corollary 4.8 of [4] implies $\operatorname{Sh} F X_{\alpha}=\operatorname{Sh} F Y_{\alpha}$, and hence by Theorem 4.1, $\mathrm{Sh}_{p} F^{*} X=\mathrm{Sh}_{p} F^{*} Y$.

Note. In the version of this paper presented at the 1978 Topology Conference in Warsaw, it was claimed that the hypothesis of Theorem 4.3 implies also that $\mathrm{Sh}_{p} \alpha X=\mathrm{Sh}_{p} \alpha Y$. The author's argument for this has proved to be defective, and the conjecture remains unsettled.

Finally, we show that Theorem 4.1 can be used to obtain a proper shape version of a result due to Y. Kodama ([9], Theorem 2), without the restriction that the spaces involved be finite dimensional.

LEMMA 4.4. If each component of $X$ is compact, then $X$ is the topological sum of compact subspaces.

Proof. By Theorem 1.1, $\mathscr{C}_{X}\left(=q^{\mathscr{C}_{X}}\right)$ is upper semicontinuous. Hence the projection map $p: X \rightarrow Q X\left(=X / \mathscr{C}_{X}\right)$ is closed and since each point-inverse under $p$ is compact, $p$ is a compact mapping (i.e., the inverse of any compact set is compact). Moreover, by Lemma 2.4, $X=\bigoplus\left\{X_{\alpha} \mid \alpha \in A\right\}$ with $Q X_{\alpha}$ compact for each $\alpha$. Since $X_{\alpha}=$ 
$p^{-1}\left(Q X_{\alpha}\right), X_{\alpha}$ is compact.

Theorem 4.5. If $\operatorname{Sh}_{p} X=\operatorname{Sh}_{p} Y$ and each component of $X$ is compact, then each component of $Y$ is compact and there is a homeomorphism $1: Q X \rightarrow Q Y$ such that for each locally compact subset $F$ of $Q X, \mathrm{Sh}_{p} p^{-1}(F)=\mathrm{Sh}_{q} q^{-1}(\Lambda(F))$, where $p: X \rightarrow Q X$ and $q: Y \rightarrow Q Y$ are the projection maps.

Proof. By Lemma 4.4, $X=\bigoplus\left\{X_{\alpha} \mid \alpha \in A\right\}$ with each $X_{\alpha}$ compact and hence by Theorem 4.1, $Y=\bigoplus\left\{Y_{\alpha} \mid \alpha \in A\right\}$ with $\operatorname{Sh}_{p} X_{\alpha}=\operatorname{Sh}_{p} Y_{\alpha}$ for each $\alpha$; since $X_{\alpha}$ is compact, $Y$ is compact ([4], p. 172) and therefore Sh $X_{\alpha}=\operatorname{Sh} Y_{\alpha}$ by Theorem 3.15 of [4]. Hence by Theorem 2.2 of [1], for each $\alpha \in A$ there is a homeomorphism $\Lambda_{\alpha}: Q X_{\alpha} \rightarrow Q Y_{\alpha}$ such that for every compact subset $K_{\alpha}$ of $Q X_{\alpha}, \operatorname{Sh} p^{-1}\left(K_{\alpha}\right)=\operatorname{Sh} q^{-1}\left(\Lambda\left(K_{\alpha}\right)\right)$. Let $\Lambda: Q X \rightarrow Q Y$ be the combination of the $\Lambda_{\alpha}$ 's(i.e., $\Lambda(a)=\Lambda_{\alpha}(a)$ if $a \in X_{\alpha}$ ). If $F$ is a locally compact subset of $Q X$ and for each $\alpha, F_{\alpha}=$ $F \cap Q X_{\alpha}$, then $F_{\alpha}$ is a locally compact subset of $Q X$. The argument for Lemma 2.3 of [1], using Theorem 4.1 in in place of Theorem 4.2 of [7], shows that $\operatorname{Sh}_{p} p^{-1}\left(F_{\alpha}\right)=\operatorname{Sh}_{p} q^{-1}\left(\Lambda_{\alpha}\left(F_{\alpha}\right)\right)$. Since $p^{-1}(F)=\bigoplus\left\{p^{-1}\left(F_{\alpha}\right) \mid \alpha \in A\right\}$ and $q^{-1}(\Lambda(F))=\bigoplus\left\{q^{-1}\left(\Lambda_{\alpha}\left(F_{\alpha}\right)\right) \mid \alpha \in A\right\}$, it follows from Theorem 4.1 that $\operatorname{Sh}_{p} p^{-1}(F)=\operatorname{Sh}_{p} q^{-1}(\Lambda(F))$.

\section{REFERENCES}

1. B. J. Ball, Shapes of saturated subsets of compacta, Coll. Math., 29 (1974), 241-246.

2. — Proper shape retracts, Fund. Math., 89 (1975), 178-189.

3. - Alternative approaches to proper shape theory, Studies in Topology (Proceedings of the 1974 Charlotte Topology Conference), Academic Press, 1975, 1-27.

4. B. J. Ball and R. B. Sher, A theory of proper shape for locally compact metric spaces, Fund. Math., 86 (1974), 163-192.

5. J. Dugundji, Topology, Allyn and Bacon, 1970.

6. H. Freudenthal, Neuaufbau der Endentheorie, Ann. of Math., 43 (1942), 261-279.

7. S. Godlewski and S. Nowak, On two notions of shape, Bull. Acad. Polon. Sci. Sér. Sci. Math. Astron. Phys., 20 (1972), 387-393.

8. J. Isbell, Uniform Spaces, Mathematica Surveys, No. 12, Amer. Math. Soc., 1964.

9. Y. Kodama, Decomposition spaces and shape in the sense of Fox, Fund, Math., 97 (1977), 199-208.

10. S. Mardešić, Shapes for topological spaces, General Topology and Appl., 3 (1973), 265-282.

11. K. Morita, On bicompactifications of semibicompact spaces, Sci. Rep. Tokyo Bunrika Daigaku, Sec. A, 4, 94 (1952), 221-229.

12. R. L. Wilder, A point set which has no true quasi-components, and which becomes connected upon the addition of a single point, Bull. Amer. Math. Soc., 33 (1927), 423427.

Received January 30, 1979. Presented at the 1978 Conference on Geometric Topology in Warsaw, and summarized in the proceedings of that conference, under the title Shapes of component-compactifications.

UNIVERsity OF Georgia

Athens, GA 30602 



\section{PACIFIC JOURNAL OF MATHEMATICS}

\section{EDITORS}

DONALD BABBITT (Managing Editor)

University of California

Los Angeles, CA 90024

HUGO RossI

University of Utah

Salt Lake City, UT 84112

C. C. Moore and ANDrew OGG

University of California

Berkeley, CA 94720
J. DUGUNDJI

Department of Mathematics University of Southern California Los Angeles, CA 90007

R. FINN and J. MILGRAM

Stanford University

Stanford, CA 94305

\section{ASSOCIATE EDITORS}
E. F. BeCKENBACH
B. H. NeumanN
F. WOLF
K. YOSHIDA

\section{SUPPORTING INSTITUTIONS}

UNIVERSITY OF BRITISH COLUMBIA CALIFORNIA INSTITUTE OF TECHNOLOGY UNIVERSITY OF CALIFORNIA MONTANA STATE UNIVERSITY UNIVERSITY OF NEVADA, RENO NEW MEXICO STATE UNIVERSITY OREGON STATE UNIVERSITY UNIVERSITY OF OREGON

\author{
UNIVERSITY OF SOUTHERN CALIFORNIA \\ STANFORD UNIVERSITY \\ UNIVERSITY OF HAWAII \\ UNIVERSITY OF TOKYO \\ UNIVERSITY OF UTAH \\ WASHINGTON STATE UNIVERSITY \\ UNIVERSITY OF · WASHINGTON
}

The Supporting Institutions listed above contribute to the cost of publication of this Journal, but they are not owners or publishers and have no responsibility for its content or policies.

Mathematical papers intended for publication in the Pacific Journal of Mathematics should be in typed form or offset-reproduced, (not dittoed), double spaced with large margins. Please do not use built up fractions in the text of the manuscript. However, you may use them in the displayed equations. Underline Greek letters in red, German in green, and script in blue. The first paragraph or two must be capable of being used separately as a synopsis of the entire paper. Please propose a heading for the odd numbered pages of less than 35 characters. Manuscripts, in triplicate, may be sent to any one of the editors. Please classify according to the scheme of Math. Reviews, Index to Vol. 39. Supply name and address of author to whom proofs should be sent. All other communications should be addressed to the managing editor, or Elaine Barth, University of California, Los Angeles, California, 90024.

50 reprints to each author are provided free for each article, only if page charges have been substantially paid. Additional copies may be obtained at cost in multiples of 50 .

The Pacific Journal of Mathematics is issued monthly as of January 1966. Regular subscription rate: $\$ 84.00$ a year (6 Vols., 12 issues). Special rate: $\$ 42.00$ a year to individual members of supporting institutions.

Subscriptions, orders for numbers issued in the last three calendar years, and changes of address should be sent to Pacific Journal of Mathematics, P.O. Box 969, Carmel Valley, CA 93924, U.S.A. Older back numbers obtainable from Kraus Periodicals Co., Route 100, Millwood, NY 10546.

PUBLISHED BY PACIFIC JOURNAL OF MATHEMATICS, A NON-PROFIT CORPORATION

Printed at Kokusai Bunken Insatsusha (International Academic Printing Co., Ltd.). 8-8, 3-chome, Takadanobaba, Shinjuku-ku, Tokyo 160, Japan.

Copyright (C) 1979 by Pacific Journal of Mathematics Manufactured and first issued in Japan 


\section{Pacific Journal of Mathematics}

\section{Vol. 84, No. $2 \quad$ June, 1979}

Somesh Chandra Bagchi and Alladi Sitaram, Spherical mean periodic

functions on semisimple Lie groups ........................ 241

Billy Joe Ball, Quasicompactifications and shape theory............. 251

Maureen A. Bardwell, The o-primitive components of a regular ordered permutation group ................................ 261

Peter W. Bates and James R. Ward, Periodic solutions of higher order

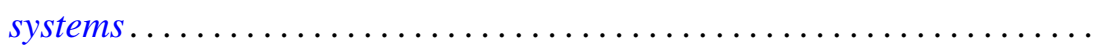

Jeroen Bruijning, A characterization of dimension of topological spaces by totally bounded pseudometrics......................... 283

Thomas Farmer, On the reduction of certain degenerate principal series

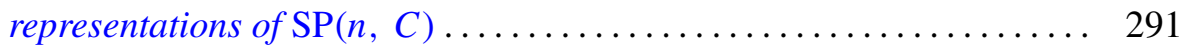

Richard P. Jerrard and Mark D. Meyerson, Homotopy with m-functions . . . . 305

James Edgar Keesling and Sibe Mardesic, A shape fibration with fibers of

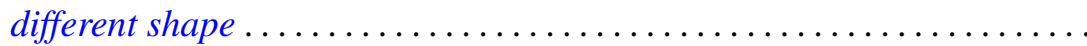

Guy Loupias, Cohomology over Banach crossed products. Application to bounded derivations and crossed homomorphisms ...............

Rainer Löwen, Symmetric planes ........................ 367

Alan L. T. Paterson, Amenable groups for which every topological left

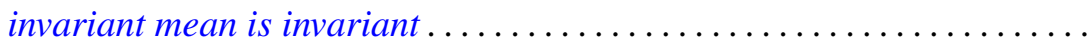

Jack Ray Porter and R. Grant Woods, Ultra-Hausdorff H-closed extensions

Calvin R. Putnam, Operators satisfying a $G_{1}$ condition .

Melvin Gordon Rothenberg and Jonathan David Sondow, Nonlinear smooth representations of compact Lie groups..............

Werner Rupp, Riesz-presentation of additive and $\sigma$-additive set-valued measures.

A. M. Russell, A commutative Banach algebra of functions of generalized variation

Judith D. Sally, Superregular sequences

Patrick Shanahan, On the signature of Grassmannians . . 